\title{
CONCORDANCIA ENTRE LÁMINAS VIRTUALES Y CONVENCIONALES EN CITOLOGÍA CERVICOVAGINAL
}

Diana Ibeth Tovar Díaz*, Karen Viviana Vega Duarte*, María del Pilar Archila, MD**, Carol Constanza Córdoba ***, Diana Patricia Miranda Cortés***

\section{Resumen}

Las láminas virtuales se presentan como una nueva técnica y herramienta en la interpretación de la CCV. Este proyecto de investigación busca establecer la concordancia existente entre el diagnóstico hecho a través de LV y el realizado en LC. Se contó con la participación de cuatro patólogos y dos citólogos, quienes analizaron 98 láminas por los dos métodos. Para el análisis de las LV se diseñó el programa informático kadiview, donde se encuentran las imágenes fotodigitales de los campos más representativos de cada LC y los datos clínicos de la paciente. El coeficiente de concordancia kappa para la clasificación general de negativo y positivo para neoplasia en los diagnósticos convencional vs. virtual fue $\mathbf{0 . 6 3}$, indicando una buena concordancia. El coeficiente kappa obtenido al analizar las categorías diagnósticas dentro de lo positivo para neoplasia, fue de 0.55 representando una concordancia moderada. Además, mediante una encuesta se estableció que el programa kadiview puede llegar a ser una herramienta muy útil para el diagnóstico en LV debido a sus características de presentación de fotos y datos clínicos. Todo lo anterior permite establecer que las LV pueden ser un método alternativo y muy útil en el diagnóstico acertado de CCV, utilizando un programa como kadiview.

Palabras clave: términos MeSH: citología, láminas; términos no MeSH: citología cervicovaginal, láminas convencionales, láminas virtuales, concordancia, kappa.

Abreviaturas: CCV, citología cervicovaginal; $L C$, láminas convencionales; $L V$, láminas virtuales.

\section{Introducción}

La citología virtual ha sido objeto de múltiples investigaciones que pretenden dar a conocer sus ventajas, desventajas y así mismo su utilidad diagnóstica. Entre estos estudios se destaca el de Gagnon y colaboradores, ${ }^{1}$ en el cual se buscaba determinar la habilidad de los profesionales en citología para hacer un diagnóstico sobre LV y luego compararlos

Fecha recibido: octubre 26 de 2006 - Fecha aceptado: diciembre 26 de 2006

* Estudiantes VI semestre de Citohistología, Fundación Universitaria de Ciencias de la Salud.

** Jefe del Servicio de Patología del Hospital de San José y profesora asociada de la Fundación Universitaria de Ciencias de la Salud.

*** Citohistotecnólogas de la Fundación Universitaria de Ciencias de la Salud. con los diagnósticos hechos a través de LC o en vidrio. A los participantes entre los que se contaban 52 patólogos y 59 citólogos, se les suministraron dos sistemas de diagnóstico: el primero consistió en láminas microscópicas convencionales, que podían ser analizadas en los equipos con los cuales ya se encontraban familiarizados (microscopios de luz) y el otro Cytoview $\mathrm{II}^{\circledR}$, compuesto por una computadora en la cual se almacenaban las LV capturadas de las microscópicas. Al final se realizó una comparación entre los dos procedimientos y se estableció que las LV pueden ser una ayuda diagnóstica para la CCV. 

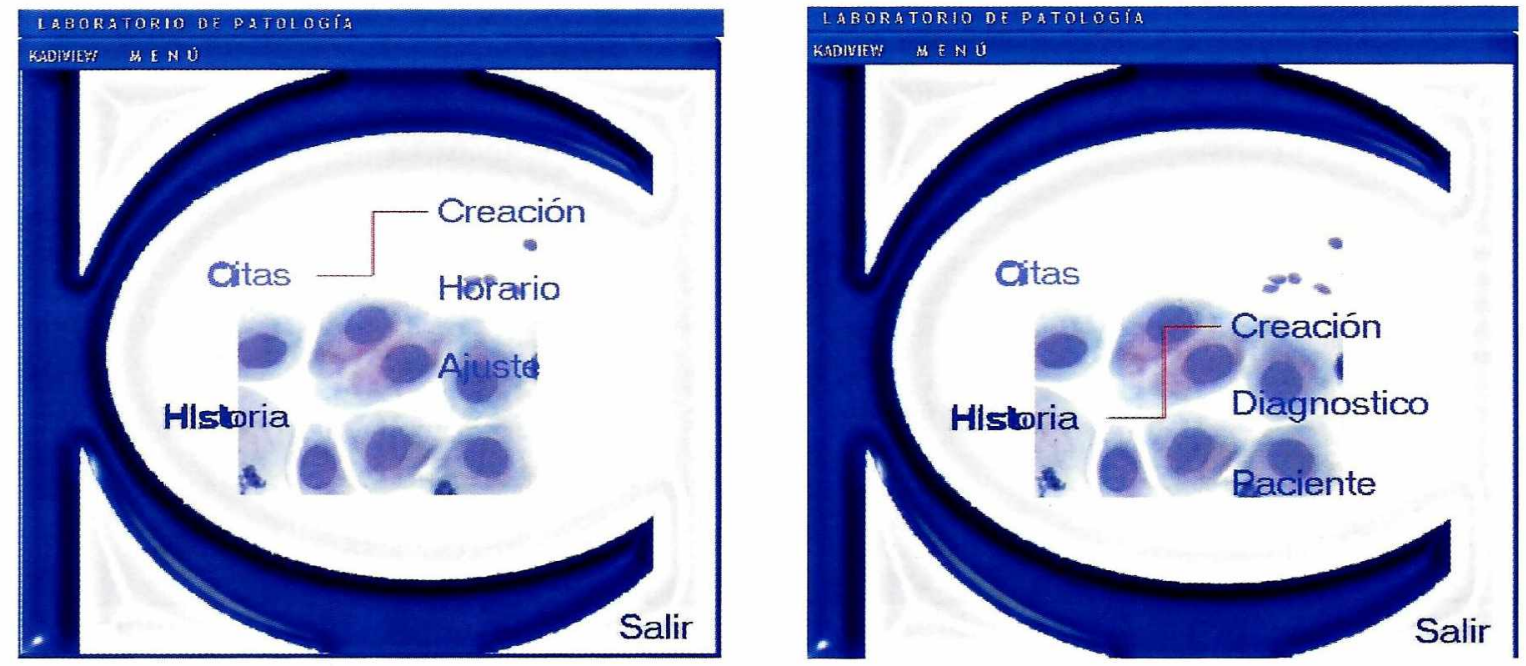

Figura I. Programa kadiview. Menú principal donde se despliega el submenú de citas e historia. En el primero se maneja el ingreso de la paciente; en el de historia se observa el caso virtual.

Continuando con la revisión se encontró que en el Instituto Trentino di Cultura en compañía del Instituto per la Ricerca Scientifica e Tecnológica (ITCirst), de Povo Trento, Italia, presentaron un nuevo método para manejar procedimientos histológicos y citológicos creando un caso virtual, el cual estaba compuesto por una colección de imágenes digitales que representaban una lámina histológica o citológica completa, con todos los niveles de amplificación y los datos clínicos más relevantes. Luego el caso podía verse en una computadora por medio de un programa que permitía seleccionar los campos más apropiados y examinarlos a diferentes amplificaciones. Su conclusión fue que comparados con las láminas en vidrio, los casos virtuales tienen varias ventajas que nacen de su naturaleza digital y pueden ser considerados una plataforma común para una amplia gama de aplicaciones como teleconsulta, educación, investigación, control de la calidad y pruebas de eficiencia. ${ }^{2}$

Resultados similares fueron descritos por el doctor Alberto M. Marchevsky y su equipo del departamento de patología del Centro Médico Cedars-Sinai en los Ángeles, California, ${ }^{3}$ y por el Centro de Control y Prevención de la Enfermedad en Georgia, EUA. ${ }^{4}$ Este proyecto de investigación pretende establecer la concordancia entre la CCV convencional y la citología virtual mediante la utilización del programa kadiview.

\section{Métodos}

A las 7.493 láminas de CCV procesadas en el laboratorio de citología de la Fundación Universitaria de Ciencias de la Salud en el período correspondiente a junio de 2005 a febrero de 2006, se les aplicaron los siguientes criterios de inclusión: buena fijación y coloración, así como aquellas con menos del 75\% de inflamación, hemorragia y citólisis.

El tamaño de la muestra calculado fue de 98 láminas, 15 negativas y 83 positivas. Se seleccionaron los campos significativos y se fotografiaron utilizando una cámara para microscopía de luz marca Javelin ${ }^{\circledR}$, para así conformar el caso virtual, el cual se instaló en kadiview. ${ }^{5}$ Este es un programa informático que permite tener acceso a los datos clínicos de las pacientes que son relevantes para la emisión de un diagnóstico en CCV, acompañados de las fotografías de los campos representativos de la LC. Estos, en conjunto, forman los casos virtuales.

A cada participante, entre los que se contaban cuatro patólogos y dos citólogos, se le suministraron noventa y ocho (98) láminas convencionales y virtuales con su respectivo protocolo para que dieran su diagnóstico. Además, ejecutaron el programa kadiview y luego lo evaluaron a través de una encuesta. Esta 

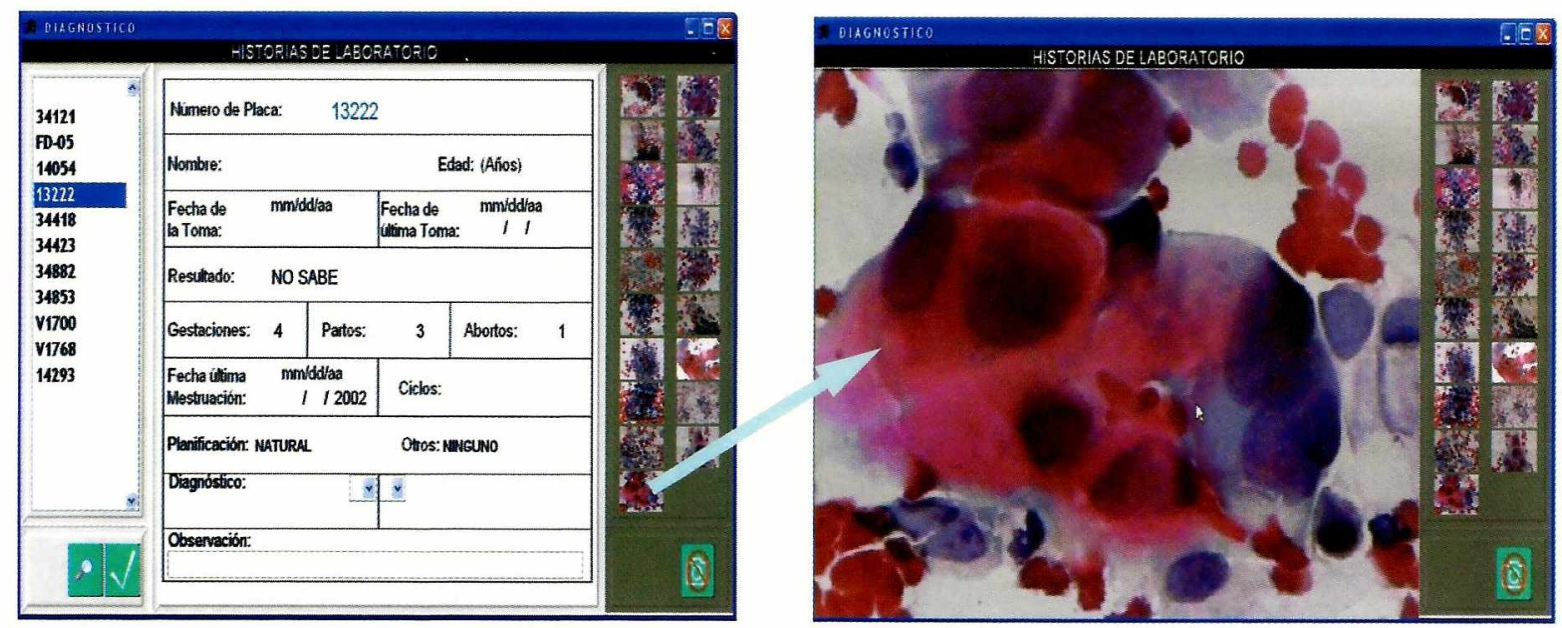

Figura. 2. Programa Kadiview: caso virtual, se observan los datos clínicos de la paciente y la galería de fotografías que forman la lámina virtual.

estaba compuesta por once preguntas cuyo objetivo era valorar el grado de aceptación, dificultades y manejo para diagnóstico de CCV virtual.

Por último, se compararon los resultados y se calculó el coeficiente kappa a través del programa estadístico Epi-info. Esto con el fin de establecer la utilidad diagnóstica del programa virtual kadiview para generar diagnósticos de CCV acertados.

Para controlar el sesgo de recuerdo de los casos por parte de los participantes (patólogos y citólogos), se dejaron intervalos mínimo de una semana entre la revisión de las LC y las LV. Otro sesgo controlado fue el del instrumento de medición por manejo inadecuado del kadiview, para lo cual se hizo una inducción.

\section{Resultados}

El número total de observaciones fue de 588, que corresponde al tamaño de muestra (98 láminas) por los seis participantes. En la Tabla 1 se muestra la concordancia de la clasificación general de negativo o positivo para malignidad.

El coeficiente kappa para clasificación general fue de 0.6311 indicando una buena concordancia dentro del índice de Landis y Koch. La Tabla 2 refleja la concordancia entre las diferentes categorías diagnósticas; se resalta que la mayor concordancia se presentó en la categoría diagnóstica LSIL, seguida de la categoría atipias escamosas. El coeficiente kappa para esta tabla fue de 0.55 representando una fuerza de concordancia moderada

La encuesta realizada para evaluar el programa kadiview y su utilidad diagnóstica reveló los siguientes resultados: sólo uno de los seis participantes había hecho diagnósticos a través de imágenes fotodigitales. Al preguntar si la selección de los casos fue acertada, cinco de los seis participantes pensaron que si lo era; kadiview cumplió con las expectativas de todos los participantes, quienes concordaron en que los datos suministrados eran suficientes para hacer un diagnóstico acertado. Las opiniones estaban divididas en cuanto a la cantidad de fotos necesaria para el diagnóstico, el 50\% opinó que se necesitaban más fotos, sobre todo para el diagnóstico de las atípias escamosas. Acerca de la inducción recibida antes de manejar el programa kadiview, cinco de los seis participantes consideraron que fue excelente (Figura 3), lo que se reflejó en que cuatro de los participantes opinaron que es muy fácil manejar el programa. En cuanto a la utilidad diagnóstica del kadiview para su labor profesional, cinco juzgaron que es excelente (Figura 4).

Las Figuras 5 y 6 representan la calificación que los participantes dieron a la calidad de las fotografías respecto al tamaño y resolución. Ellos estimaron que el orden de presentación de los datos de la historia clínica era excelente. 


\section{Discusión}

En la actualidad, la interpretación de la CCV se hace a través de la lectura directa de las LC mediante un microscopio de luz. En países como el nuestro, la dificultad para estudiar de los casos en los cuales la CCV resulta sospechosa o positiva para neoplasia es grande, en especial en aquellos lugares de tipo rural, de difícil acceso o restringido, pues el personal calificado se limita a las grandes ciudades, convirtiéndose en una de las problemáticas más sentidas en el sistema de salud. Por esta razón, las nuevas tecnologías se presentan como una herramienta importante para acercar a estos grupos aislados a establecimientos de salud de mayor nivel.

Surge por lo tanto, un innovador método que permitiría a los patólogos y citólogos una mayor reproducibilidad de los diferentes diagnósticos, que a su vez se reflejaría en un tratamiento oportuno que mejorará la calidad de vida de las pacientes. De igual manera, con fines docentes kadiview sirve como plataforma de aprendizaje para estudiantes de patología y citología, así como para los cursos de actualización dirigidos a los profesionales en estas áreas. También podría utilizarse como prueba de proeficiencia para patólogos y citólogos.

El programa informático kadiview sirve como medio para resolver estas necesidades, en tanto que permite el acceso a los datos clínicos relevantes de la paciente
TABLA I. Concordancia de la clasificación general de negativo y positivo para neoplasia. Diagnóstico convencional vs. diagnóstico virtual

\begin{tabular}{|c|c|c|c|}
\hline \multicolumn{4}{|c|}{ Dx. virtual } \\
\hline $\begin{array}{c}\text { Dx. } \\
\text { convencional }\end{array}$ & NEGATIVO & POSITIVO & TOTAL \\
\hline NEGATIVO & 63 & 30 & 93 \\
\hline POSITIVO & 27 & 468 & 495 \\
\hline TOTAL & 90 & 498 & 588 \\
\hline
\end{tabular}

y a las fotografías de los campos representativos de la CCV convencional, lo que en conjunto formaría el caso virtual al cual el usuario patólogo o citólogo tendría acceso para dar su diagnóstico. Este programa fue considerado por los participantes como una excelente ayuda en el estudio de la CCV.

Aunque nuestra hipótesis de investigación suponía que el coeficiente kappa era de 0.7 , en nuestro estudio fue de 0.63 con respecto a los resultados en la clasificación general de negativo y positivo para neoplasia y 0.55 en las diferentes categorías diagnósticas. La disminución pudo ser consecuencia de la falta de experiencia en el diagnóstico sobre LV por parte de los participantes, ${ }^{5,6}$ ya que solo uno de ellos lo había hecho. Otros factores revelados en la encuesta fueron la falta de fondo en algunas fotografías, la baja resolución y en algunos casos la cantidad de estas no era suficiente.

TABLA 2. Concordancia entre las diferentes categorías diagnósticas de negativo y positivo para neoplasia. Diagnóstico convencional vs. diagnóstico virtual

Dx. virtual

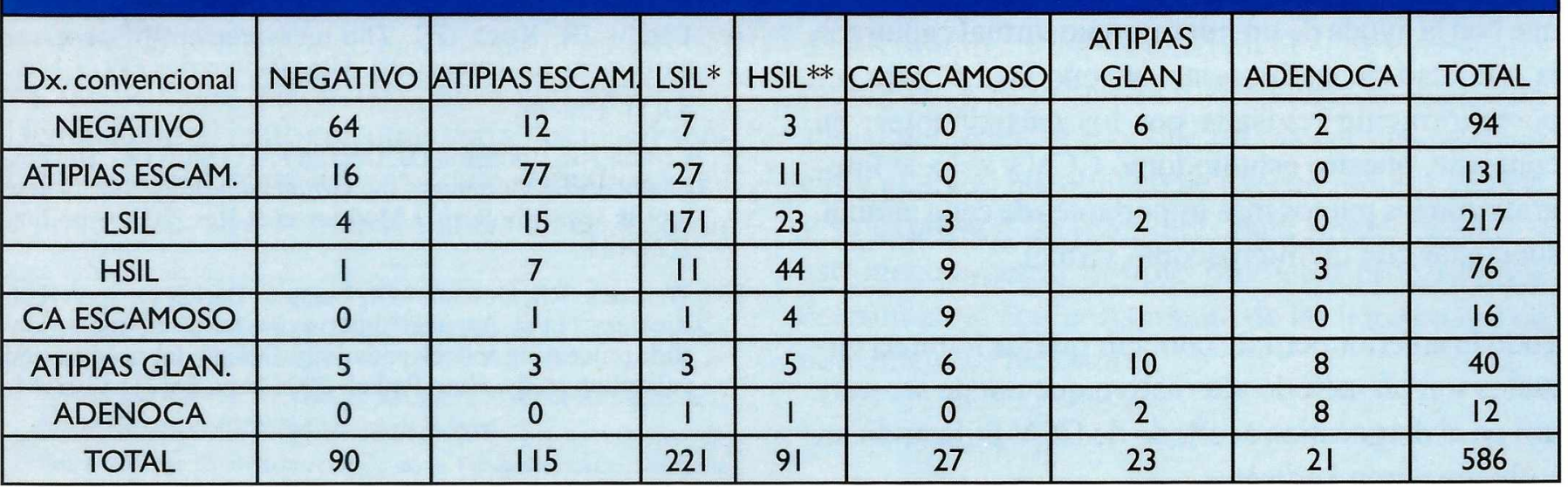




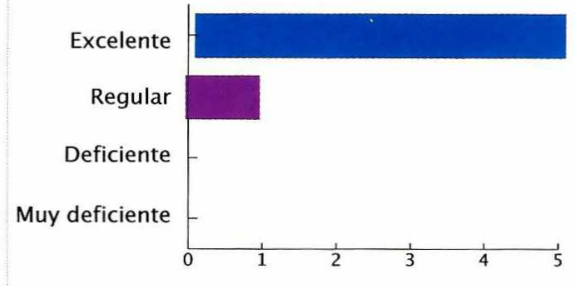

Figura 3.Inducción a kadiview.

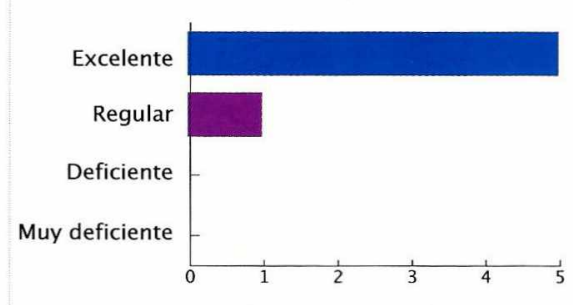

Figura 5. Tamaño de fotos.

Al comparar el coeficiente kappa 0.63 conseguido en este estudio con el que obtuvo Costello y colaboradores $^{7}$ sobre el desarrollo y evaluación de láminas virtuales en patología $($ kappa $=0.75)$ se observa que es menor; esto se explica porque en este último se examinaron biopsias y no citologías. Además, los 17 observadores podían revisar casi la totalidad de la biopsia, ya que realizaban como promedio 22 observaciones por caso, mientras que en nuestro estudio el número de fotografías era 20 y no cubrían la totalidad del extendido citológico sino los campos más representativos. En nuestro estudio los participantes eran seis y tuvieron que examinar 98 casos, tanto en LC como LV mientras que el estudio de Costello eran 17 participantes y solo examinaban 10 láminas. Weinstein y colaboradores ${ }^{8}$ determinaron en su estudio un coeficiente kappa 0.96 utilizando un programa para microscopía virtual llamado DMetrix que con la ayuda de un microscopio virtual capturaba la totalidad de las biopsias de seno en LC para ser posteriormente revisada por los participantes; en contraste, nuestro estudio tomó CCV y solo se fotografiaron los puntos más importantes de cada lámina, sin contar con un microscopio virtual.

Todo lo anterior permite concluir que las láminas virtuales son un método alternativo que puede ser muy útil en el diagnóstico acertado de CCV utilizando un software como kadiview.

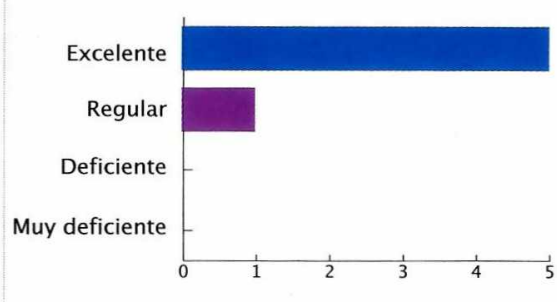

Figura 4. Utilidad de kadiview.

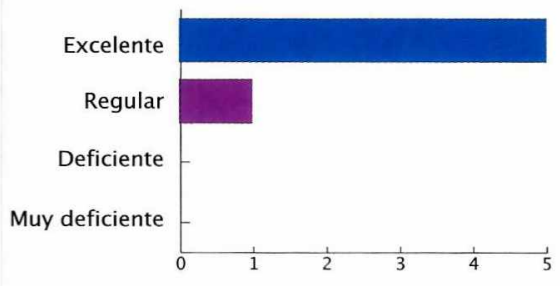

Figura 6. Resolución de las fotos.

\section{Referencias}

1. Gagnon M, Inhorn S, Hancock J, Keller B, Carpenter D, Merlin T, et al. Comparison of cytology proficiency testing: glass slides vs. virtual slides.Acta Cytol. 2004 Nov-Dec; 48(6): 788-94.

2. Demichelis F, Barbareschi M, Dalla Palma P, Forti S. The virtual case: a new method to completely digitize cytological and histological slides. Virchows Arch. 2002 Aug; 441(2):159-64. Epub 2001 Dec 12.

3. Marchevsky AM, Wan Y, Thomas P, Krishnan L, EvansSimon H, Haber H. Virtual microscopy as a tool for proficiency testing in cytopathology: a model using multiple digital images of Papanicolau tests. Arch Pathol Lab Med. 2003 Oct; 127(10):1320-4.

4. Taylor RN, Gagnon M, Lange J, Lee T, Draut R, Kujawski E. Cyto View: a prototype computer image-based Papanicolaou smear proficiency test. Acta Cytol. 1999 Nov-Dec; 43(6):1045-51.

5. Cicchetti, D.V, Fleiss, J.L. Comparison of the null distribution of weighted kappa and the C ordinal statistic. Appl Psychol Meas. 1977; 1: 195-201.

6. Landis JR, Koch GG. The measurement of observer agreement for categorical data. Biometrics. 1977 Mar; 33(1): 159-74.

7. Costello SS, Johnston DJ, Dervan PA, O'Shea DG. Development and evaluation of the virtual pathology slide: a new tool in telepathology. J Med Internet Res. 2003 Apr-Jun; 5(2): e11.

8. Weinstein RS, Descour MR, Liang C, Barker G, Scott KM, Richter L, et al. An array microscope for ultrarapid virtual slide processing and telepathology. Design, fabrication, and validation study. Hum Pathol. 2004 Nov; 35(11):1303-14. 